\title{
In vitro postantibiotic effects of tomopenem (CS-023) against Staphylococcus aureus and Pseudomonas aeruginosa
}

\author{
Takanori Tomozawa, Chika Sugihara, Masayo Kakuta, Kiyoshi Sugihara \\ and Tetsufumi Koga
}

Correspondence

Tetsufumi Koga

koga.tetsufumi.zj@daiichisankyo.

co.jp

Received 30 November 2009

Accepted 18 January 2010

\author{
Biological Research Laboratories IV, Daiichi Sankyo Co. Ltd, 1-2-58 Hiromachi, Shinagawa-ku, \\ Tokyo 140-8710, Japan
}

\begin{abstract}
The postantibiotic effect (PAE) of tomopenem was determined after a $2 \mathrm{~h}$ exposure of two strains of meticillin-susceptible and meticillin-resistant Staphylococcus aureus (MSSA and MRSA), and imipenem-susceptible and imipenem-resistant Pseudomonas aeruginosa, to tenfold the respective MIC. The PAEs on MSSA and $P$. aeruginosa were approximately $1 \mathrm{~h}$ and they were comparable to those of meropenem. The PAE on MRSA was 1.5 to $3 \mathrm{~h}$, equal to or longer than those of vancomycin. The PAEs of tomopenem not only were found for MRSA, but also were present in the imipenem-resistant $P$. aeruginosa tested.
\end{abstract}

\section{INTRODUCTION}

Tomopenem (formerly CS-023) is a novel $1 \beta$-methyl carbapenem with a broad spectrum of activity against clinically important Gram-positive and Gram-negative bacteria, including meticillin-resistant Staphylococcus aureus (MRSA) and Pseudomonas aeruginosa. We have previously reported that the favourable features of tomopenem are as follows: (i) better antibacterial activity against $P$. aeruginosa than imipenem and meropenem, and in particular, potent activity against imipenem-resistant $P$. aeruginosa (Koga et al., 2005); (ii) correlation between the potent antibacterial activity against MRSA and a high affinity for penicillinbinding protein 2a (Koga et al., 2008); and (iii) a longer elimination half-life than imipenem and meropenem in clinical studies (Shibayama et al., 2006).

The purpose of this study was to determine the in vitro postantibiotic effects (PAEs) of tomopenem on meticillinsusceptible $S$. aureus (MSSA), MRSA, imipenem-susceptible $P$. aeruginosa, and imipenem-resistant $P$. aeruginosa and to compare them with the PAEs of the antibiotics routinely used in clinical practice to treat infections caused by these pathogens.

\section{METHODS}

Antibiotics. Tomopenem was synthesized at Daiichi Sankyo Research Laboratories, Tokyo, Japan. Pure samples of imipenem (Banyu Pharmaceutical), meropenem (Dainippon Sumitomo Pharma) and ceftazidime (GlaxoSmithKline) were extracted at
Daiichi Sankyo from commercially supplied compounds. Vancomycin hydrochloride was purchased from Sigma-Aldrich.

Bacterial strains. MRSA 12404, MRSA 12412, and imipenemresistant $P$. aeruginosa 12435 and 12438 (metallo- $\beta$-lactamase-negative strains) were isolated in Japanese hospitals in 1999. The reference strains used in this study were stock cultures from our laboratory.

Susceptibility tests. The MICs were determined by a standard microdilution broth method (CLSI, 2006). Mueller-Hinton broth (Becton Dickinson) containing $25 \mathrm{mg} \mathrm{Ca}^{2+} 1^{-1}$ and $12.5 \mathrm{mg} \mathrm{Mg}^{2+} \mathrm{l}^{-1}$ [cation-adjusted Mueller-Hinton broth (CAMHB)] was used. The MIC was defined as the lowest concentration of the compound that completely inhibited the viable growth of the organism in the microdilution wells. The MIC determination was performed in duplicate.

PAE. The PAE was determined by the viable plate count method (Craig \& Gudmundsson, 1996). The organisms were freshly subcultured and incubated at $37{ }^{\circ} \mathrm{C}$ with shaking to obtain a final inoculum of approximately $5 \times 10^{6}$ c.f.u. $\mathrm{ml}^{-1}$. Bacterial suspensions of each organism were then exposed to $10 \times$ MIC of each compound for $2 \mathrm{~h}$. A culture containing no compound was included as a growth control. At the end of the exposure period, the cultures were diluted 1000 -fold with CAMHB in order to remove the compounds. Viable counts were determined for all cultures at this time (time 0 ). The cultures were placed back in a shaker, with sampling performed every hour on heart infusion agar plates. The 10-fold serially diluted samples (diluted with CAMHB) were pipetted into a sterile Petri plate and $10 \mathrm{ml}$ melted heart infusion agar was added. Three independent tests were carried out for each bacterial strain.

For calculation of the PAE ( $\mathrm{min}$ ), the PAE was defined as $T-C$, where $T$ represents the time required for the count in the compound-exposed culture to increase by $1 \log _{10}$ above the count observed at time 0 , and where $C$ is the corresponding time for the control culture. Each PAE was calculated independently. The mean and its $95 \%$ confidence interval for each PAE were determined using SAS system release 8.2. 
Statistical analysis. A $t$ test was performed with the hypothesis that the mean PAE of the three experiments was equal to 0 for each compound. A $P$ value of less than 0.05 was considered statistically significant.

\section{RESULTS AND DISCUSSION}

Tomopenem has enhanced activity against MRSA and $P$. aeruginosa, and improved pharmacokinetic properties in comparison with commercially available carbapenems such as imipenem and meropenem. Carbapenems induce a consistent PAE both in vitro and in vivo against both Gram-positive and Gram-negative bacteria, including $P$. aeruginosa (Odenholt et al., 1989). However, very few data have been reported regarding the PAE of carbapenems on
MRSA and imipenem-resistant $P$. aeruginosa. Therefore, in this study we determined the PAEs on these pathogens. Since the duration of the PAE increased until a maximum response was obtained, usually at a concentration that was approximately $10 \times$ MIC (Vogelman \& Craig, 1985; Zhanel et al., 1991), we chose this concentration. Table 1 shows the PAEs of tomopenem against $S$. aureus and $P$. aeruginosa. For MSSA, the PAEs of tomopenem were almost the same as those of imipenem, meropenem and ceftazidime. For MRSA 12412, the PAEs of tomopenem were longer than those of vancomycin. MacGowan et al. (2008) reported that tomopenem had a superior effect compared to vancomycin in an in vitro pharmacokinetic model of MRSA infection, possibly because of its longer PAE. For $P$. aeruginosa ATCC 27853, the PAE of imipenem in the

Table 1. PAE of drugs on $S$. aureus and $P$. aeruginosa

A $t$ test was performed for the hypothesis that the mean PAE was equal to 0 .

\begin{tabular}{|c|c|c|c|c|c|}
\hline Organism and drug & $\operatorname{MIC}\left(\mathrm{mg} \mathrm{l}^{-1}\right)$ & PAE (min) & SD & $\begin{array}{c}95 \% \text { confidence } \\
\text { interval }\end{array}$ & $P$ value \\
\hline \multicolumn{6}{|c|}{ S. aureus ATCC 6538P } \\
\hline Imipenem & 0.008 & 64 & 3 & $56-72$ & 0.0008 \\
\hline Meropenem & 0.06 & 56 & 12 & $26-87$ & 0.0156 \\
\hline Ceftazidime & 1 & 54 & 18 & $10-98$ & 0.0336 \\
\hline Tomopenem & 0.12 & 55 & 14 & $21-89$ & 0.0199 \\
\hline Imipenem & 0.016 & 59 & 1 & $55-62$ & 0.0002 \\
\hline Meropenem & 0.12 & 56 & 4 & $46-66$ & 0.0018 \\
\hline Ceftazidime & 4 & 48 & 8 & $29-68$ & 0.0086 \\
\hline \multicolumn{6}{|l|}{ MRSA 12404} \\
\hline Tomopenem & 4 & 101 & 11 & $74-128$ & 0.0039 \\
\hline Vancomycin & 1 & 110 & 15 & $72-148$ & 0.0063 \\
\hline \multicolumn{6}{|c|}{ P. aeruginosa ATCC 15692} \\
\hline Tomopenem & 0.5 & 66 & 8 & $47-85$ & 0.0044 \\
\hline Imipenem & 1 & 100 & 18 & $55-145$ & 0.0108 \\
\hline Meropenem & 1 & 60 & 12 & $30-90$ & 0.0133 \\
\hline Ceftazidime & 2 & -41 & 7 & $-60--23$ & 0.0105 \\
\hline \multicolumn{6}{|c|}{ P. aeruginosa ATCC 27853} \\
\hline Tomopenem & 0.5 & 73 & 17 & $32-114$ & 0.0168 \\
\hline Imipenem & 2 & 96 & 23 & $39-152$ & 0.0181 \\
\hline Meropenem & 0.5 & 64 & 18 & $19-110$ & 0.0257 \\
\hline Ceftazidime & 2 & -45 & 14 & $-81--10$ & 0.0320 \\
\hline \multicolumn{6}{|c|}{ IPM-resistant $P$. aeruginosa $12435^{\star}$} \\
\hline
\end{tabular}

${ }^{\star}$ Imipenem-resistant $P$. aeruginosa (MIC of imipenem $\geqslant 16 \mathrm{mg} \mathrm{l}^{-1}$ ). 
current study is similar to that in another report (Odenholt et al., 1989). The PAEs of tomopenem for P. aeruginosa were almost the same as those of meropenem, and were slightly shorter than those of imipenem. Ceftazidime showed negative PAEs against two strains of $P$. aeruginosa. Ceftazidime showed a high affinity to PBP 3 and caused a filament formation on Gram-negative bacteria, which therefore produced negative PAEs. On the contrary, Gould et al. (1989) expressed the notion that an affinity to PBP 2 is related to the induction of PAE. Since tomopenem binds to PBP 2 in $P$. aeruginosa with high affinity that results in bulge formations on cells (Koga et al., 2009), it showed positive PAEs, as did the other carbapenems. For the imipenem-resistant $P$. aeruginosa tested, the PAEs of tomopenem were almost the same as those of meropenem, and the PAEs on imipenemsusceptible and -resistant isolates were no different. The reason for this is not clear; however, the PAEs may not be different between susceptible and resistant strains on the basis of MIC. Since there have been reports about various types of MRSA (Hanssen \& Ericson Solid, 2006) and carbapenem-resistant P. aeruginosa (Quale et al., 2006), further study is required on tomopenem against additional strains to provide more detailed information about PAEs.

Tomopenem has a longer half-life (1.7 h) in comparison with imipenem (Drusano \& Standiford, 1985) and meropenem (Bax et al., 1989), and the protein binding value of tomopenem in human serum is low $(9.5 \%$; Shibayama et al., 2007), similar to those of imipenem and meropenem. Since we have shown that the activity of tomopenem against $S$. aureus and $P$. aeruginosa was minimally affected by twofold with the addition of horse serum (Ohya et al., 2000), it is suggested that serum would have little impact on the PAEs. A single $1400 \mathrm{mg}$ dose in healthy Caucasian volunteers (Shibayama et al., 2006) and a $1500 \mathrm{mg}$ dose in healthy Japanese volunteers (Sesoko et al., 2006) yielded maximum concentrations in serum of $102 \pm 15$ and $122 \pm 14 \mathrm{mg} \mathrm{l}^{-1}$, respectively. At these doses, the concentrations in serum could exceed $40 \mathrm{mg} \mathrm{l}^{-1}$ in about $2 \mathrm{~h}$. Since the MIC of tomopenem against MRSA and the imipenem-resistant $P$. aeruginosa tested was $4 \mathrm{mg} \mathrm{l}^{-1}$, tomopenem may help prevent the regrowth of these bacteria if the drug levels drop below the MIC between doses for these organisms. In addition to the PAEs, the effect of subinhibitory concentrations on bacteria previously treated (the postantibiotic subMIC effect) or not (subMIC effect) is another important parameter (Odenholt-Tornqvist et al., 1991). The postantibiotic subMIC effect represents the time interval that includes the PAE plus the additional time during which growth is suppressed by subMIC concentrations. The postantibiotic subMIC effect and subMIC effect reflect serum drug levels that may exist between antibiotic dosing intervals and the success of discontinuous dosing regimens. These parameters should also be considered in the event that an optimal dosage regimen for tomopenem can be determined.

\section{REFERENCES}

Bax, R. P., Bastain, W., Featherstone, A., Wilkinson, D. M., Hutchison, M. \& Haworth, S. J. (1989). The pharmacokinetics of meropenem in volunteers. J Antimicrob Chemother 24 (Suppl. A), 311-320.

CLSI (2006). Methods for Dilution Antimicrobial Susceptibility Tests for Bacteria that Grow Aerobically, 7th edn, approved standard M7-A7. Wayne, PA: Clinical and Laboratory Standards Institute.

Craig, W. A. \& Gudmundsson, S. (1996). The postantibiotic effect. In Antibiotics in Laboratory Medicine, 4th edn, pp. 296-329. Edited by V. Lorian. Baltimore, MD: Williams \& Wilkins.

Drusano, G. L. \& Standiford, H. C. (1985). Pharmacokinetic profile of imipenem/cilastatin in normal volunteers. Am J Med 78 (Suppl. 6A), 47-53.

Gould, I. M., Jason, A. C. \& Milne, K. (1989). Use of the Malthus microbial growth analyser to study the post antibiotic effect of antibiotics. J Antimicrob Chemother 24, 523-531.

Hanssen, A. M. \& Ericson Solid, J. U. (2006). SCCmec in staphylococci: genes on the move. FEMS Immunol Med Microbiol 46, 8-20.

Koga, T., Abe, T., Inoue, H., Takenouchi, T., Kitayama, A., Yoshida, T., Masuda, N., Sugihara, C., Kakuta, M. \& other authors (2005). In vitro and in vivo antibacterial activity of CS-023 (RO4908463), a novel parenteral carbapenem. Antimicrob Agents Chemother 49, 3239-3250.

Koga, T., Masuda, N., Kakuta, M., Namba, E., Sugihara, C. \& Fukuoka, T. (2008). Potent in vitro activity of tomopenem (CS-023) against methicillin-resistant Staphylococcus aureus and Pseudomonas aeruginosa. Antimicrob Agents Chemother 52, 2849-2854.

Koga, T., Sugihara, C., Kakuta, M., Masuda, N., Namba, E. \& Fukuoka, T. (2009). Affinity of tomopenem (CS-023) for penicillinbinding proteins in Staphylococcus aureus, Escherichia coli, and Pseudomonas aeruginosa. Antimicrob Agents Chemother 53, 12381241.

MacGowan, A. P., Bowker, K. E. \& Noel, A. R. (2008). Pharmacodynamics of the antibacterial effect and emergence of resistance to tomopenem, formerly RO4908463/CS-023, in an in vitro pharmacokinetic model of Staphylococcus aureus infection. Antimicrob Agents Chemother 52, 1401-1406.

Odenholt, I., Isaksson, B., Nilsson, L. \& Cars, O. (1989). Postantibiotic and bactericidal effect of imipenem against Pseudomonas aeruginosa. Eur J Clin Microbiol Infect Dis 8, 136-141.

Odenholt-Tornqvist, I., Löwdin, E. \& Cars, O. (1991). Pharmacodynamic effects of subinhibitory concentrations of $\beta$-lactam antibiotics in vitro. Antimicrob Agents Chemother 35, 1834-1839.

Ohya, S., Abe, T., Ishii, C., Kakuta, M., Masuda, N., Utsui, Y. \& Kuwahara, S. (2000). R-115685, a novel parenteral carbapenem: in vitro antibacterial activity. In Program and Abstracts of the 40th Interscience Conference on Antimicrobial Agents and Chemotherapy, abstract F-1230. Toronto, Canada: American Society for Microbiology.

Quale, J., Bratu, S., Gupta, J. \& Landman, D. (2006). Interplay of efflux system, ampC, and oprD expression in carbapenem resistance of Pseudomonas aeruginosa clinical isolates. Antimicrob Agents Chemother 50, 1633-1641.

Sesoko, S., Morita, K., Tajima, N. \& Nakashima, M. (2006). Safety, tolerability, and pharmacokinetics (PK) of CS-023, a new parenteral carbapenem, in healthy Japanese male volunteers. In Program and Abstracts of the 42nd Interscience Conference on Antimicrobial Agents and Chemotherapy, abstract A-1941. San Francisco: American Society for Microbiology.

Shibayama, T., Matsushita, Y., Hirota, T., Ikeda, T. \& Kuwahara, S. (2006). Pharmacokinetics of CS-023 (RO4908463), a novel parenteral 
carbapenem, in healthy male Caucasian volunteers. Antimicrob Agents Chemother 50, 4186-4188.

Shibayama, T., Matsushita, Y., Kurihara, A., Hirota, T. \& Ikeda, T. (2007)

Prediction of pharmacokinetics of CS-023 (RO4908463), a novel parenteral carbapenem antibiotic, in humans using animal data. Xenobiotica 37, 91-102.
Vogelman, B. S. \& Craig, W. A. (1985). Postantibiotic effects. J Antimicrob Chemother 15 (Suppl. A), 37-46.

Zhanel, G. G., Hoban, D. J. \& Harding, G. K. M. (1991). The postantibiotic effect: a review of in vitro and in vivo data. DICP 25 , 153-163. 